\title{
Atlas-Based Segmentation and Tracking of 3D Cardiac MR Images Using Non-rigid Registration
}

\author{
M. Lorenzo-Valdés ${ }^{1}$, G.I. Sanchez-Ortiz ${ }^{1}$, R. Mohiaddin ${ }^{2}$, and D. Rueckert ${ }^{1}$ \\ 1 Visual Information Processing Group, Department of Computing, \\ Imperial College of Science, Technology, and Medicine, \\ 180 Queen's Gate, London SW7 2BZ, United Kingdom \\ 2 Royal Bromptom and Harefield NHS Trust, Sydney Street, \\ London, United Kingdom
}

\begin{abstract}
We propose a novel method for fully automated segmentation and tracking of the myocardium and left and right ventricles (LV and RV) using 4D MR images. The method uses non-rigid registration to elastically deform a cardiac atlas built automatically from 14 normal subjects. The registration yields robust performance and is particularly suitable for processing a sequence of $3 \mathrm{D}$ images in a cardiac cycle. Transformations are calculated to obtain the deformations between images in a sequence. The registration algorithm aligns the cardiac atlas to a subject specific atlas of the sequence generated with the transformations. The method relates images spatially and temporally and is suitable for measuring regional motion and deformation, as well as for labelling and tracking specific regions of the heart. In this work experiments for the registration, segmentation and tracking of a cardiac cycle are presented on nine MRI data sets. Validation against manual segmentations and computation of the correlation between manual and automatic tracking and segmentation on $1413 \mathrm{D}$ volumes were calculated. Results show that the procedure can accurately track the left ventricle ( $\mathrm{r}=0.99)$, myocardium ( $\mathrm{r}=0.98)$ and right ventricle $(\mathrm{r}=0.96)$. Results for segmentation are also obtained for left ventricle $(\mathrm{r}=0.92)$, myocardium $(\mathrm{r}=0.82)$ and right ventricle $(\mathrm{r}=0.90)$.
\end{abstract}

\section{Introduction}

The diagnosis and treatment monitoring of cardiac malfunction has improved with the advent of cardiac imaging techniques that provide 4D images of the heart by acquiring a sequence of $3 \mathrm{D}$ images throughout the cardiac cycle. An accurate identification of the borders of the structures to be analysed is needed in order to extract physiologically meaningful quantitative information from the images. Applications of cardiac segmentation include the calculation of volume and mass, blood ejection fraction, analysis of contraction and wall motion as well as the 3D visualisation of cardiac anatomy [1]. Segmentation needs to be automated in order to be clinically valuable, because when done manually it is very time consuming and partly subjective.

While good results for wall motion and left ventricular (LV) function have been shown in imaging modalities like SPECT or 3D echocardiography [2]3], segmentation and tracking of other important anatomical structures like the right ventricle (RV) and myocardium are limited by the imaging methodologies, and the low contrast and signal 
to noise ratio of the images. Recently, steady state free precession cine MR imaging with balanced gradients, known as TrueFISP [4], has been shown to be less susceptible to artifacts caused by slow flow and to provide significantly enhanced blood-myocardial contrast in comparison with conventional gradient echo image sequences. These TrueFISP sequences allow a better delineation of the cardiac borders and have led to an increased interest in automatic segmentation techniques for 4D MR images.

Several approaches have been proposed for the automatic segmentation of cardiac structures in MR images (for a review see [5]). Recently, a number of techniques which are based on the use of a model or an atlas have been proposed [6,7]. In these approaches the atlas is used to incorporate a-priori information which enables the use of both intensity and spatial information during the segmentation process. In particular active shape and appearance models [8] have shown promising results, where segmentation is achieved by minimisation of the difference in appearance between the model and the object of interest [9]. Most of these techniques work only for 2D even though extensions to 3D have been recently proposed [10]. A common problem of these techniques is the fact that the applicability of active appearance models is limited to the MR imaging sequence used for training since the intensity appearance is an explicit part of the statistical model. In addition, they do not exploit the relationship between time frames.

In this paper we propose a fully automated method for segmentation and tracking of the myocardium and ventricles which uses a non-rigid registration algorithm based on free-form deformations (FFDs) to register a model in the form of a cardiac atlas to a cine sequence of 3D MR volumes. Our approach to segmentation and tracking is registration-based and therefore fundamentally different from previous approaches to tracking of the heart using FFDs [11]. Registration-based segmentation is often used in brain segmentation [12]. In this work we are using registration to align an atlas of the heart with 3D cardiac MR images taking advantage of the spatial and temporal correlation of the images in a cardiac sequence. In the following we explain the registration algorithm, the construction of the atlas, and developed methodology in detail. Results are assessed by comparing to manual segmentations on nine subjects.

\section{Non-rigid Registration}

The purpose of image registration is to map points in one image to their corresponding point in another image [13]. In our case we are interested in finding corresponding points between a 3D atlas of the heart and 3D MR image as well as between different time frames in a sequence of 3D MR images. Figure 1 illustrates deformations of a 3D atlas after it has been registered to a cardiac MR image. To model these deformations we are using a non-rigid registration technique which has been previously used for a number of different applications [1415]. This algorithm uses a combined transformation model that consists of a global and local transformation:

$$
\mathbf{T}(x, y, z)=\mathbf{T}_{\text {global }}(x, y, z)+\mathbf{T}_{\text {local }}(x, y, z)
$$

The global motion can be described by an affine transformation which allows scaling, translation, rotation and shearing of the shape. The local deformations are represented using a free-form deformation (FFD) model based on B-splines. The basic idea of FFDs is to deform an object by manipulating an underlying mesh of control points. The resulting 


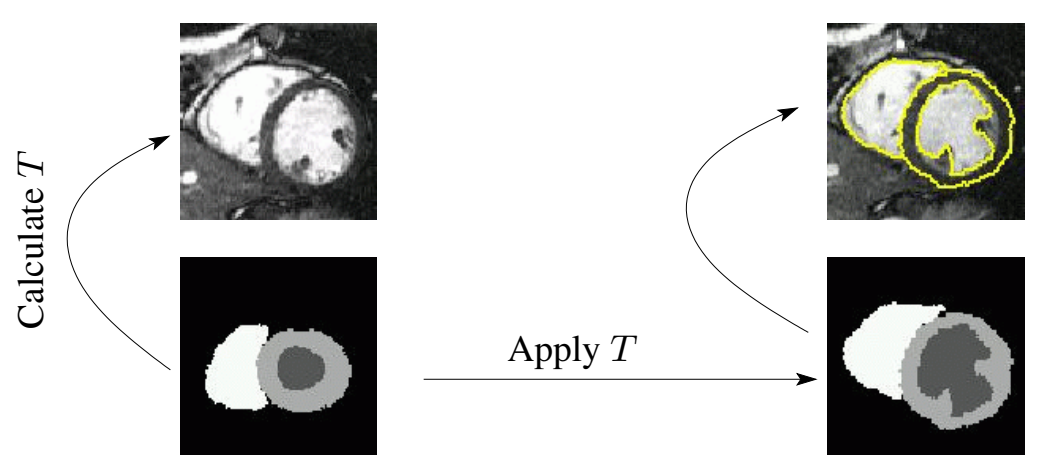

Fig. 1. Registration of a 3D cardiac atlas in form of a labelled image to a 3D MR image of the heart.

deformation controls the shape of the $3 \mathrm{D}$ object and can be written as the $3 \mathrm{D}$ tensor product of the familiar 1D cubic B-splines,

$$
\mathbf{T}_{\text {local }}(x, y, z)=\sum_{t=0}^{3} \sum_{m=0}^{3} \sum_{n=0}^{3} B_{l}(u) B_{m}(v) B_{n}(w) \phi_{i+l, j+m, k+n}
$$

where $\phi$ denotes the control points which parameterise the transformation; $u, v$ and $w$ denote the lattice coordinates of $x, y$ and $z$ and $B_{l}$ is the $l$-th B-spline basis function.

To relate the 3D cardiac atlas in form of a labelled image with a cardiac MR image, a measure of alignment between them is necessary. Information-theoretic similarity measures such as mutual information and normalised mutual information have been frequently used for the registration of multi-modal images. Since normalised mutual information only measures the statistical dependencies between the intensity distributions in both images but not the dependencies between the intensities directly, it can be used to align binary or labelled images (e.g. a cardiac atlas) with intensity images (e.g. cardiac MR images). For this reason we use normalised mutual information [16] as a measure of alignment between the 3D cardiac atlas and the cardiac MR image. For two images $A$ and $B$, normalised mutual information $I$ is defined as:

$$
I(A, B)=\frac{H(A)+H(B)}{H(A, B)}
$$

where $H(A), H(B)$ are the marginal entropies of images $A, B$ and $H(A, B)$ denotes the joint entropy between images $A$ and $B$.

\section{Atlas-Based Segmentation of the Heart}

The key idea of the proposed technique is to reduce the problem of segmentation of the entire sequence of 3D MR images to the one of segmenting the end-diastolic time frame of this sequence (in our case the end-diastolic frame corresponds to the first frame of the sequence). In this section we describe how an automatic segmentation of the heart in the end-diastolic time frame can be obtained by non-rigid registration of a populationspecific atlas to a subject-specific atlas corresponding to the end-diastolic time frame. 

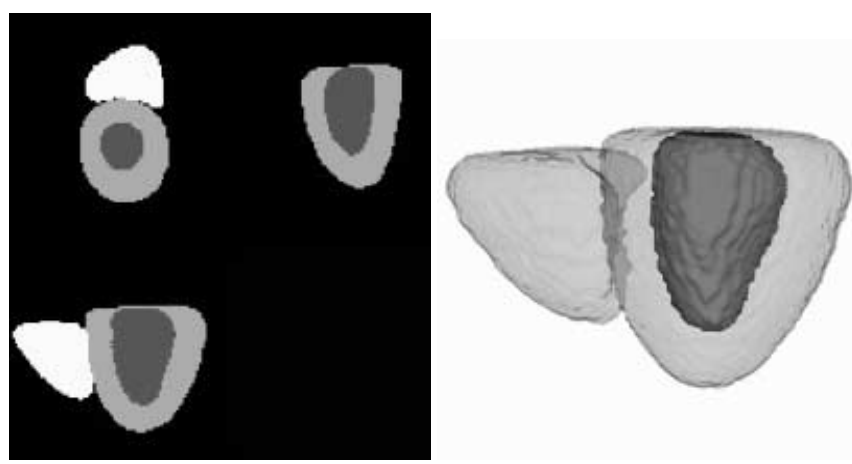

Fig. 2. The orthogonal views of the cardiac atlas (left) and the corresponding surfaces of the myocardium, left and right ventricle (right). The atlas has been constructed from 14 volunteers as described in [17.18].

In the following we describe the construction of the population- and subject-specific atlases.

\subsection{Construction of a Population-Specific Atlas}

Fourteen normal adults were scanned on a 1.5 Tesla MR scanner (Philips ACS-NT, PowerTrak 6000 Gradient System) using an ECG-triggered Echo Planar Imaging (FFEEPI) sequence. Cine acquisitions consisting of eight to ten short-axis slices of the heart and eighteen to twenty phases of the cardiac cycle were performed. From the acquired temporal sequence of each volunteer, the end diastolic frame was manually segmented. Using these segmented images we have constructed a labelled 3D atlas [17 18] containing the myocardium and the left and right ventricles. Figure 2 shows the labelled atlas and the corresponding epi- and endocardial surfaces. It is important to note that the subjects used for the construction of the atlas are different from the subjects used for the validation of the proposed segmentation technique.

\subsection{Construction of a Subject-Specific Atlas}

As mentioned previously, a segmentation of the end-diastolic time frame is achieved by performing non-rigid registration between the population-specific atlas and the enddiastolic time frame of the 3D MR image sequence. The accurate and robust segmentation of this frame is crucial in order to allow the propagation of this segmentation throughout the entire sequence of 3D MR images. In order to reduce noise and obtain better contrast between the anatomical structures we have constructed a subject-specific intensity atlas of the heart corresponding to the end-diastolic time frame. This atlas has been calculated by registering all time frames to the end-diastolic time frame. This can be achieved by using the transformations as illustrated in the diagram of Fig. 3 After applying these transformations $\mathbf{T}$ the transformed images were averaged to produce an average intensity atlas which describes the cardiac anatomy at end diastole. Note the improved contrast and definition of the borders of the average intensity atlas compared to the original MR image. 


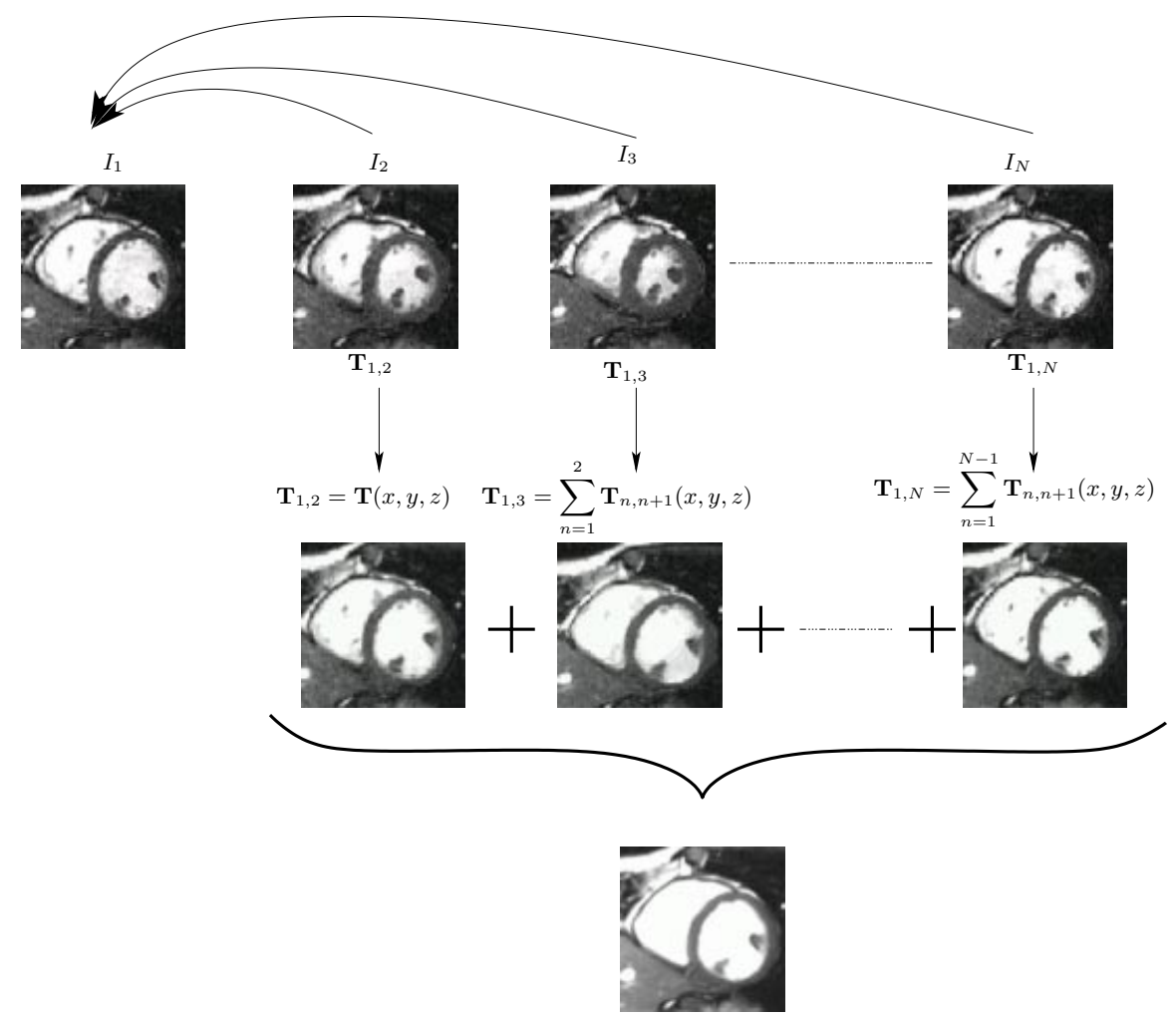

Fig. 3. Procedure to obtain a subject-specific atlas. $I_{n}$ is the $3 \mathrm{D}$ MR volume at time $n$ and $\mathbf{T}_{1, n}$ is the transformation that maps a point in the cardiac anatomy at time $n$ into the corresponding point at time 1 . The intensities are averaged to obtain the atlas.

\section{Atlas-Based Tracking of the Heart}

If the correspondences between the end-diastolic frame and all subsequent time frames are known, we can propagate the segmentation of the end-diastolic frame through the entire cardiac cycle. To calculate the correspondences within this sequence we are using an extension of the non-rigid registration technique to multi-level FFDs proposed by Schnabel et al. [19]. In this model the deformation between two consecutive time frames $n$ and $n+1$ is represented as a single-resolution FFD, $\mathbf{T}_{n+1, n}$ and is defined by the local transformation of eq. (2) which maps a point in time frame $n$ into the corresponding point in time frame $n+1$. The deformation between the end-diastolic time frame $(n=1)$ and time frame $N$ is defined by a multi-level FFD, $\mathbf{T}_{N, 1}$, which is the sum of the local deformations throughout the sequence, i.e.:

$$
\mathbf{T}_{N, 1}(x, y, z)=\sum_{n=1}^{N-1} \mathbf{T}_{n+1, n}(x, y, z)
$$

Each non-rigid registration between the end-diastolic time frame and time frame $N$ yields a transformation $\mathbf{T}_{N, 1}$ that was used as initial transformation estimate $\mathbf{T}_{N+1,1}$ for the 


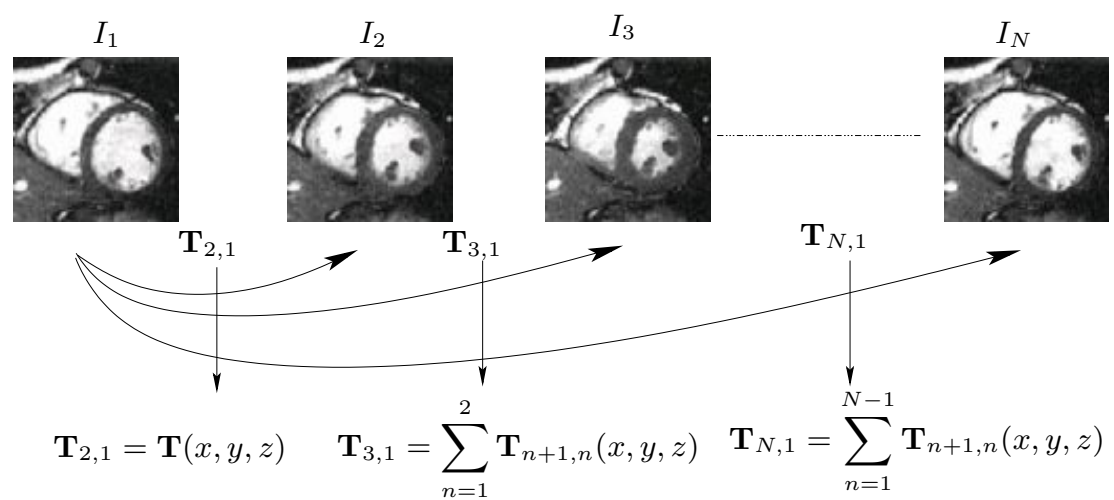

Fig. 4. Tracking of a cardiac sequence. $I_{n}$ is the $3 \mathrm{D}$ MR volume at time $n$ and $\mathbf{T}_{n+1, n}$ is the transformation that maps a point in the cardiac anatomy at time $n$ into the corresponding point at time $n+1$.

registration of time frame $N+1$. Therefore, The resulting transformations contained an accumulation of the transformations obtained starting from the end-diastolic frame and thus allowing to track the heart through the entire cardiac sequence (see Figure 4).

\section{Results and Discussion}

In order to assess the performance of the automatic segmentation method, results were compared against those obtained by manually segmenting nine 3D image sequences for the left ventricle (LV), right ventricle (RV) and myocardium. The images were acquired at the Royal Brompton Hospital, London, UK from nine healthy volunteers. Each image sequence comprises between 10 and 17 time frames, involving a total of 141 volumetric images. The images were acquired using a Siemens Sonata 1.5T scanner, with a TrueFisp sequence and $256 \times 256 \times 10$ voxels. The field of view ranged between $300-350 \mathrm{~mm}$ and thickness of slices was $10 \mathrm{~mm}$.

The volumes of the ventricles and myocardium were calculated and linear regression analysis was used to compare the manually and the automatically segmented images. Two different experiments were performed: The first experiment assessed the tracking of the heart in a cardiac sequence while the second experiment evaluated the fully automatic segmentation and tracking of the heart using the atlas. To assess the tracking, the transformations between different time frames were applied to the manual segmentation of the end-diastolic frame instead of the atlas. The volumes of the LV, myocardium and RV were calculated. Results in Figure 5 yield a very good correlation for all the structures $(\mathrm{LV}=0.99$, myocardium $=0.98, \mathrm{RV}=0.96$ ). In the second experiment the enddiastolic time frame has been segmented by deforming the population-specific atlas to the subject-specific atlas (corresponding to the end-diastolic time frame). Results in Figure 6 show the correlation between manual and automatic segmentation ( $\mathrm{LV}=0.94$, myocardium $=0.83, \mathrm{RV}=0.92$ ). An example of automatic segmentation is shown in Figure 7. where the original image and its corresponding automatic segmentation are displayed.

\section{Conclusions and Future Work}

The presented approach enables the automatic segmentation of 4D cardiac MR images taking advantage of the temporal relation between images in order to identify the main 

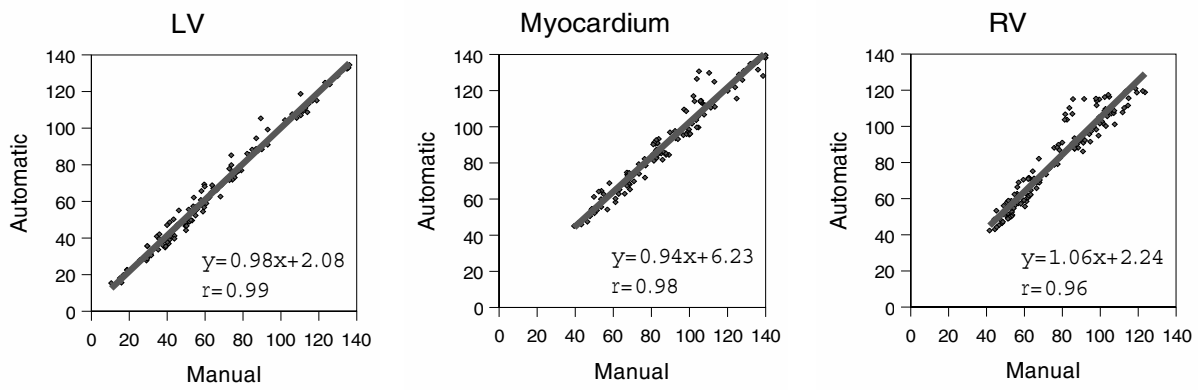

Fig. 5. Comparison of the volumes obtained by manual and automatic segmentation using the manual segmentation of the end-diastolic time frame to assess the tracking throughout the sequence (volumes are given in $\mathrm{cm}^{3}$ ).
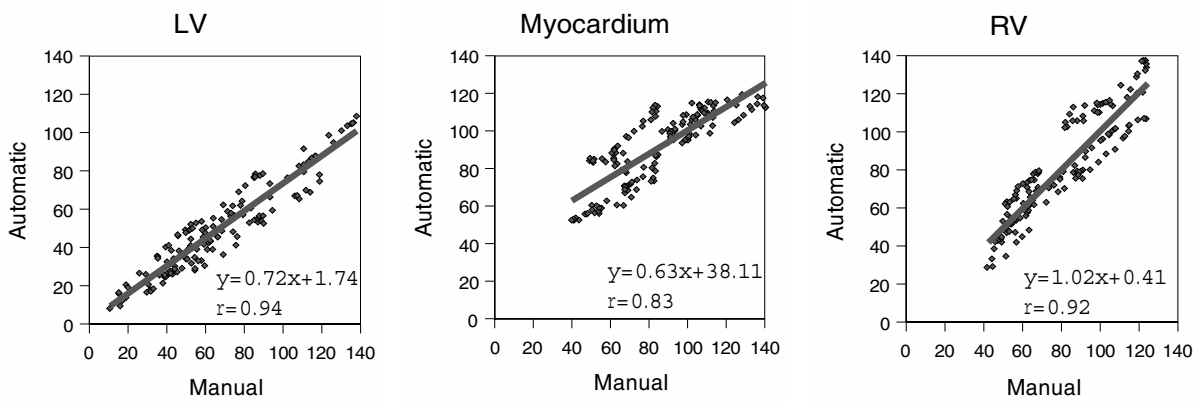

Fig. 6. Comparison of the volumes obtained by manual and automatic segmentation using the atlas-based segmentation of the end-diastolic time frame to assess the tracking throughout the sequence (volumes are given in $\mathrm{cm}^{3}$ ).
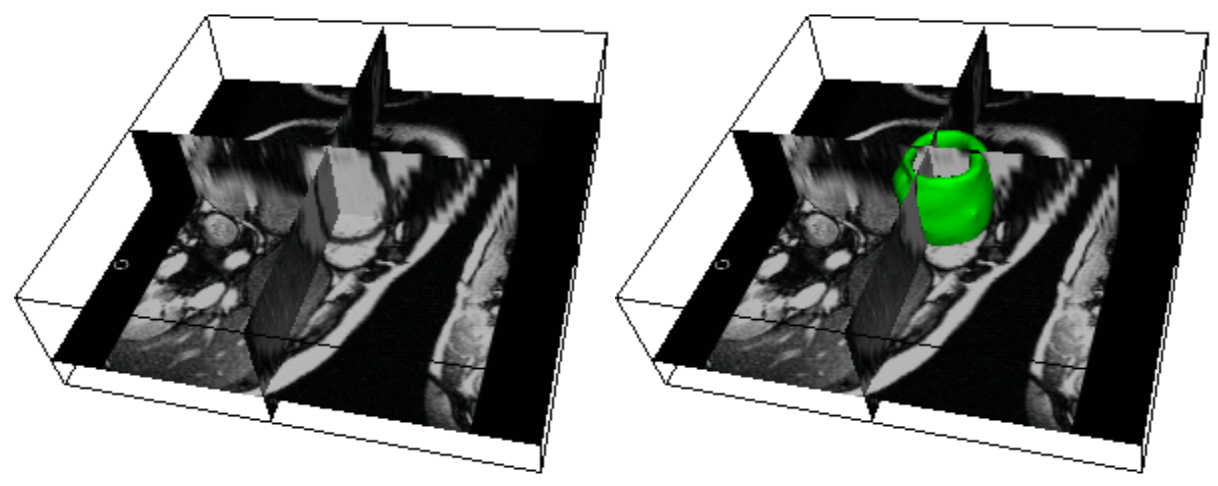

Fig. 7. Atlas-based segmentation of the end-diastolic time frame. An end-diastolic MR volume (shown on the left) with the deformed atlas (shown on the right as a green surface). An animated version of an entire cine sequence can be found at http://www.doc.ic.ac.uk/ dr/projects/animations/MICCAI 02. 
structures of the heart. Since the proposed method is atlas-based it enables the accurate and robust segmentation and tracking not only of the LV, but also of the RV and myocardium. In addition, it is independent of the image intensities in the MR images and can be applied to images acquired with other sequences (e.g. black-blood studies). The method has a major limitation, since the segmentation of the end-diastolic frame is still prone to errors, especially the segmentation of the myocardium where the contrast within other tissues is too low and might be underestimated or overestimated. These errors are propagated and they may even increase in subsequent time frames. This was reflected in the results where there were clusters corresponding to the different cardiac image sequences.

Future work will involve two essential aspects of the method presented. First, the spatial and temporal transformations computed by the registration algorithm provide the information necessary to assess regional motion and deformation, which are essential for the detection of various types of cardiac malfunction (e.g. ischaemia). Secondly, the registration of the atlas to the images also makes the method suitable for monitoring regions of interest (e.g. the septum). This can be achieved using a generic atlas for labelling specific regions of the heart and tracking them during the cardiac cycle.

\section{Acknowledgements}

M. Lorenzo-Valdés is funded by a grant from CONACyT, México. G. I. Sanchez-Ortiz is funded by EPSRC grant no. GR/R41002/01.

\section{References}

1. A. F. Frangi, W. J. Niessen, and M. A. Viergever. Three-dimensional modeling for functional analysis of cardiac images: A review. IEEE Transactions on Medical Imaging, 20(1):26-35, 2001.

2. J.P. Thirion and S. Benayoun. MyoTrack:A 3D deformation field method to measure cardiac motion from gated SPECT. In Medical Image Computing and Computer-Assisted Intervention, Lecture Notes in Computer Science, pages 697-706, Pittsburgh, USA, Oct 2000.

3. G.I. Sanchez-Ortiz, J. Declerck, J.A. Noble, and M. Mulet-Parada. Automating 3D Echocardiographic Image Analysis. In Medical Image Computing and Computer-Assisted Intervention, Lecture Notes in Computer Science, pages 687-696, Pittsburgh, USA, Oct 2000.

4. S. Plein, T.N. Bloomer, J.P. Ridgway, T.R. Jones, G.J. Bainbridge, and M.U. Sivananthan. Steady-state free precession magnetic resonance imaging of the heart: Comparison with segmented k-space gradient-echo imaging. Journal of Magnetic Resonance Imaging, 14(3):230236, 2001.

5. J. S. Suri. Computer vision, pattern recognition and image processing in left ventricle segmentation: The last 50 years. Pattern Analysis and Applications, 3(3):209-242, 2002.

6. F. Vincent, P. Clarysse, P. Croiselle, and I. Magnin. Segmentation of the heart from MR image sequences using a 3D active model. Annals of Biomedical Engineering, 28(1):S-42, 2000.

7. B.P.F. Lelieveldt, R.J. Van der Geest, R.M. Ramze, J.G. Bosch, and J.H.C. Reiber. Anatomical model matching with fuzzy implicit surfaces for segmentation of thoracic volume scans. IEEE Transactions on Medica Imaging, 18(3):218-230, 1999.

8. T. F. Cootes, G. J. Edwards, and C. J. Taylor. Active appearance models. In Proc. 5th European Conference on Computer Vision (ECCV'98), volume 2, pages 484-498, 1998. 
9. S.C. Mitchell, B.P.F. Lelieveldt, R.J. Van Der Geest, H. G. Bosch, J.H.C. Reiber, and M. Sonka. Multistage hybrid active appearance model matching: Segmentation of left and right ventricles in cardiac MR images. IEEE Transactions on Medical Imaging, 20:415-423, 2001.

10. S.C. Mitchell, B.P.F. Lelieveldt, J. G. Bosch, R. Van Der Geest, J.H.C. Reiber, and M. Sonka. Segmentation of cardiac MR volume data using 3D active appearance models. In SPIE Conference on Medical Imaging, Image Processing, page In press, 2002.

11. E. Bardinet, L. D. Cohen, and N. Ayache. Tracking and motion analysis of the left ventricle with deformable superquadratics. Medical Image Analysis, 1(2):129-149, 1996.

12. D. L. Collins, A. C. Evans, C. Holmes, and T. M. Peters. Automatic 3D segmentation of neuro-anatomical structures from MRI. In Y. Bizais et al., editor, Information Processing in Medical Imaging, pages 139-152. Kluwer Academic Publishers, 1995.

13. J. M. Fitzpatrick, D. L. G. Hill, and C. R. Maurer, Jr. Image registration. In Milan Sonka and J. Michael Fitzpatrick, editors, Handbook of Medical Imaging, volume 2, pages 447-513. SPIE Press, 2000.

14. D. Rueckert, L.I. Sonoda, C. Hayes, D.L.G. Hill, M.O. Leach, and D.J. Hawkes. Nonrigid registration using free-form deformations: Application to breast CT images. IEEE Transactions on Medical Imaging, 18(8):712-721, 1999.

15. C. R. Maurer, Jr., D. L. G. Hill, A. J. Martin, H. Liu, M. McCue, D. Rueckert, D. Lloret, W. A. Hall, R. E. Maxwell, D. J. Hawkes, and C. L. Truwit. Investigation of intraoperative brain deformation using a $1.5 \mathrm{~T}$ interventional MR system: preliminary results. IEEE Transactions on Medical Imaging, 17(5):817-825, 1998.

16. C. Studholme, D.L.G. Hill, and D.J. Hawkes. Automated three-dimensional regisration of magnetic resonance and positron emission tomography brain images by multiresolution optimization of voxel similarity measures. Medical Physics, 24(1):71-86, 1997.

17. A. F. Frangi, D. Rueckert, J. A. Schnabel, and W. J. Niessen. Automatic 3D ASM construction via atlas-based landmarking and volumetric elastic registration. In Information Processing in Medical Imaging: Proc. 17th International Conference (IPMI'01), pages 78-91, Davis, CA, July 2001.

18. A. F. Frangi, D. Rueckert, J. A. Schnabel, and W. J. Niessen. Automatic construction of multiple-object three-dimensional statistical shape models: Application to cardiac modeling. IEEE Transactions on Medical Imaging, 2001. Submitted.

19. J.A. Schnabel, D. Rueckert, M. Quist, J.M. Blackall, A.D. Castellano-Smith, T. Hartkens, G.P. Penney, W.A. Hall, H. Liu, C.L. Truwit, F.A. Gerritsen, D.L.G. Hill, and D.J. Hawkes. A generic framework for non-rigid registration based on non-uniform multi-level free-form deformations. In Medical image computing and computed-assisted intervention - MICCAI, pages 573-581, 2001. 